\title{
Advances in Research on Trust, Trusted Systems, and Digital Technologies
}

\author{
Sirkka L. Jarvenpaa \\ The University at Texas Austin \\ sirkka.jarvenpaa@mccombs.tex \\ $\underline{\text { as.edu }}$
}

\author{
Mareike Möhlmann \\ Warwick Business School \\ mareike.moehlmann@wbs.ac.uk
}

\author{
Kirsimarja Blomqvist \\ LUT University \\ $\underline{\text { Kirsimarja.blomqvist@1ut.fi }}$
}

\begin{abstract}
Within the Conference on System Sciences (HICSS), we organize for the fourth time a minitrack on Advances in Research on Trust, Trusted Systems, and Digital Technologies. Through six papers and an open discussion, the minitrack will debate when and to what degree trust matters, in what form(s), and with which consequences in the context of digital technologies, e.g., electronic markets and e-commerce, robots, artificial intelligence, and digital platforms.
\end{abstract}

\section{Introduction}

Trust is a pervasive concern, not just with new digital technologies but also with established technology systems as they are interrelated and becoming more complex. We identify need to theoretically or empirically advance our understanding of different forms of trust in organizational, inter-organizational, network, platform, and interpersonal contexts involving digital technologies.

In order to address these emerging contexts, this mini-track advances research on trust, trusted systems, and digital technologies. Some of the emerging questions discussed and debated in the minitrack include the following:

What are the various risks and vulnerabilities to trust imposed by emerging algorithmic capabilities, cloud-based platforms, complex platform infrastructures, and highly distributed peer-to-peer systems? How do new advancements in both hardware and software technologies change the way we trust and trust-related processes? What forms will digital trust and trust in digital environments take? What shapes trust and its consequences? What are the implications for trust as technologies take on capabilities with both social and moral agency? Some digital technologies may replace the trust we now have in institutions, as trust shifts from humans and central organizations to computers and anonymous decentralized organizations that know no geographic boundaries. When do trust in technology and trust in humans complement each other? For example, can trust and control complement each other in AI adoption? How do trust in humans, organizations, and technology interact with each other? How can digital platforms build swift trust? How do trust processes in digital platforms and sharing economy context differ from traditional interpersonal trust models? How does trust in humans differ from trust in technology? Is it possible that human-like systems exacerbate rather than compensate weaknesses common in trust assessments? How does blockchain technology change the way we think about trust? How does trust evolve as an interplay of trust in different trust referents, e.g. individuals, technological systems and societal institutions?

\section{Minitrack Papers}

The minitrack comprises of two sessions. The three papers to be presented in the first session address trust referents and reputation. First, the paper by Anthony Gibson, Gene Alarcon, Sarah Jessup, August Capiola has the title Do you still trust me? Effects of Personality on Changes in Trust during an Experimental Task with a Human or Robot Partner. This paper explores the effects of three dispositional variables (i.e., dispositional trust, dispositional distrust, and dispositional suspicion) on self-reported trust and suspicion perceptions towards a partner (a human or a Nao robot).

Second, the paper co-authored by Kirsimarja Blomqvist and Lisa van der Werff titled Understanding organizational stakeholder trust expectations for AIbased services, offers a case study on different organizational stakeholders' trust-related expectations 
at micro and macro-levels in AI-based service development.

Third, the paper by Timm Teubner and Maik Hesse it titled Takeaway Trust: A market data perspective on reputation portability in electronic commerce. Based on market data from a marketplace, the paper empirically investigates cross-platform reputation portability.

In the second session will comprise three papers that are exploring the foundations of trust. First, the paper by Matthias Söllner has the title Same Same But Different? A Two-Foci Perspective on Trust in Information Systems. This piece of research provides a first evaluation of a conceptual piece claiming a) that users distinguish between their trust in an IS and the provider of this IS and $b$ ) that both kinds of trust are important for the success sustainable success of IS providers.

Second, the paper co-authored by Maik Hesse, David Dann, Fabian Braesemann, Timm Teubner is Understanding the Platform Economy: Signals, Trust, and Social Interaction. The paper offers a crossplatform comparison by focusing on how platforms that offer services which vary in their degree of social interaction implement different trust mechanisms.

Third, the paper by Obi Ogbanufe, Dan Kim is titled Confirmatory Influence of Trust in E-commerce: A Data Collection Bias and Suggestion. This paper explores trust in the context of e-commerce transaction by empirically investigating the impact of trust on the intention to purchase from both successful and unsuccessful purchase cases.

\section{Discussion of Research Advances}

The papers presented at the mini-track represent a good reflection of recent advances in trust research in the IS community. All authors rely on traditional definitions for trust from interpersonal, organizational, and information systems literatures. For example, Anthony Gibson, Gene Alarcon, Sarah Jessup and August Capiola draw on work by Mayer, Davis and Schoorman (1995) when defining trust as a person's willingness to be vulnerable to another, without the ability to monitor that trustee's actions. Their study focuses on trustor's dispositional variables and they test how they differ whether the trustee is a human or robot. Ogbanufe and Kim draw adopt a 3-item Internet consumer trust measure by Kim et al (2009) referring to vendor integrity and benevolence as well as ecommerce site trustworthiness.

Yet, authors take a holistic view in studying various dispositions to trust, what kind of trust cues trustors read, and how trust is built, and refer to multiple trust referents, in multi-layered environments (e.g. platforms). For example, Mathias Söllner distinguish between different trust referents, which are trust in information system and the provider of an information system. Hesse, Dann, Braesemann and Teubner acknowledge that several trust referents may co-exist by exploring the trust building mechanisms and related trust cues provided by various platform providers vs. trust cues in the providers valued by different platform customers.

Other authors argue that trust can be built via impersonal and personal, direct and indirect, subjective and objective mechanisms at micro- and macro-levels derived from direct or indirect experiences and expectations. For example, Hesse, Dann, Braesemann and Teubner build a taxonomy for trust building cues consisting of transaction-based assessments, user profiles such as profile image and self-description, identity verifications, and implicit information such as status badges or average response time. Teubner and Hesse study reputation systems' textual and numerical aggregate transaction-based feedback as a signal for trustworthiness. Kirsimarja Blomqvist and Lisa van der Werff compare how different organizational stakeholders perceive AI trustworthiness, and what kind of trust cues they read at micro- and macro levels of analysis.

One of the developments we identify in this recent research on trust, trusted systems, and digital technologies is that while trust research has long been dominated by interpersonal trust and organizational trust, the sources, referents, and mechanisms in perceiving and building trust are becoming more and more varied, increasingly including non-human and impersonal, e.g. technological trust referents. Furthermore, research is increasingly concerned about how trust evolves in complex and multi-layered environments such as digital platforms and ecosystems.

Given these recent advances in trust research, we identify several topics that trust research in information systems management can address with potential for high theoretical and practical impact. We find it is important that researchers on trust in information systems develop theories that help to explain how emerging technologies affect individuals and 
organizations' perceptions of trust, and the trust relationships between users and emerging technologies. We urge future research to address the role of trust in the development and implementation of algorithms, e.g., functions; openness of coding, data collection and implementation of new services. Especially, in the future we would like to see more research addressing multi-level trust contexts, i.e. its evolvement across time and different trust referents. Process studies could be helpful in understanding the dynamic and changing nature of trust as well as advancing insight useful for practitioners designing and implementing complex information systems and digital technologies. Increasingly, we expect to see digital technologies augmenting human interaction and would be interested in seeing research on the interplay of digital and human trust cues. In addition, research on digital trust technologies, such as blockchain in emerging technological forms and their governance would be welcomed. Finally, we urge researchers to focus on the mechanisms and means how organizations handle users' data, e.g., privacy/integrity/security, use of the cloud, and trust in the organization.

We hope that our mini-track can provide a good venue for discussion on the papers as well as further research opportunities of the increasingly important topic of the relationship of trust and emerging technologies. 